\title{
Advancing Risk Assessment of Intermediate Risk Prostate Cancer Patients
}

\author{
Darrel Drachenberg ${ }^{1}$, Julius A. Awe ${ }^{2}$, Aline Rangel Pozzo ${ }^{2}$, Jeff Saranchuk ${ }^{1}$ and \\ Sabine Mai ${ }^{2, *(1)}$ \\ 1 Section of Urology, Department of Surgery, Manitoba Prostate Center, Cancer Care Manitoba, \\ University of Manitoba, Winnipeg, MB R3E 0V9, Canada; drach13@mymts.net (D.D.); \\ jsaranchuk@exchange.hsc.mb.ca (J.S.) \\ 2 Cell Biology, Research Institute of Hematology and Oncology, Cancer Care Manitoba, University of Manitoba, \\ Winnipeg, MB R3E 0V9, Canada; d4mostcts@yahoo.com (J.A.A.); aline.rangelpozzo@umanitoba.ca (A.R.P.) \\ * Correspondence: sabine.mai@umanitoba.ca; Tel.: +1-204-787-2135
}

Received: 6 May 2019; Accepted: 17 June 2019; Published: 20 June 2019

check for updates

\begin{abstract}
The individual risk to progression is unclear for intermediate risk prostate cancer patients. To assess their risk to progression, we examined the level of genomic instability in circulating tumor cells (CTCs) using quantitative three-dimensional (3D) telomere analysis. Data of CTCs from 65 treatment-naïve patients with biopsy-confirmed D'Amico-defined intermediate risk prostate cancer were compared to radical prostatectomy pathology results, which provided a clinical endpoint to the study and confirmed pre-operative pathology or demonstrated upgrading. Hierarchical centroid cluster analysis of 3D pre-operative CTC telomere profiling placed the patients into three subgroups with different potential risk of aggressive disease. Logistic regression modeling of the risk of progression estimated odds ratios with 95\% confidence interval (CI) and separated patients into "stable" vs. "risk of aggressive" disease. The receiver operating characteristic (ROC) curve showed an area under the curve (AUC) of 0.77, while prostate specific antigen (PSA) (AUC of 0.59) and Gleason $3+4=7$ vs. $4+3=7(p>0.6)$ were unable to predict progressive or stable disease. The data suggest that quantitative $3 \mathrm{D}$ telomere profiling of CTCs may be a potential tool for assessing a patient's prostate cancer pre-treatment risk.
\end{abstract}

Keywords: intermediate risk prostate cancer; Gleason 7; circulating tumor cells; three-dimensional (3D) imaging; 3D nucleus; quantitative 3D telomere analysis

\section{Introduction}

Prostate cancer is the second most common cancer in men and is a heterogeneous disease with both indolent and more aggressive forms. Gleason patterns and Gleason scores have long been the single most important predictors of cancer treatment prognosis. However, patients with the same Gleason score 7 can often have vastly different outcomes [1-5]. With current methods, the clinical prognostic grouping for localized prostate cancer is imprecise, with 30-50\% of patients recurring after image-guided radiotherapy or radical prostatectomy [1]. Close to $20 \%$ of intermediate-risk patients develop biochemical failure that occurs within 18 months of primary local therapy [1]. The consequence of imprecise clinical prognostic grouping is that some indolent tumors are overtreated, while more aggressive ones may mistakenly receive no or delayed treatment [6,7].

The apparent paradox in clinical prognostic grouping of patients and the inter- and intra-personal differences between patients of the same pathology grouping is linked to the level of genomic instability present in the patient's tumor: More genomic instability in individual tumor cells sets the stage for ongoing tumor cell (micro)evolution and leads to the generation of several subsets of tumor cells with distinct genetic properties. These genetic variants contribute to tumor development and progression [1-5,8,9]. 
Since tissue biopsies rarely represent the whole tumor due to inherent sampling issues, liquid biopsies become an attractive option in the assessment of a patient's tumor [10,11]. They offer the opportunity of multiple and less invasive sampling from the patient's blood and allow for the monitoring of the dynamic process of tumor evolution (for review, see [12]). Liquid biopsies have commonly focused on the analysis of circulating tumor cells (CTCs), cell-free nuclei acids (DNA and RNA) as well as exosomes; among all of these types of liquid biopsies, most work has been done with CTCs. While their limitations are linked to their rare numbers or even absence in some patients, a difficulty that is also encountered by other types of liquid biopsies, the use of CTCs in the clinic is becoming a reality $[10,13]$. Clinical validation for CTCs in metastatic-castration-resistant prostate cancer is most mature $[10,13-15]$. One prominent example is the nuclear-specific androgen receptor variant 7 (AR-V7) detection on CTCs in this patient group [16]. The presence or absence of this protein is required to inform treatment decisions for these patients [16].

Our current study focused on intermediate risk prostate cancer. This risk group is defined as Gleason $3+4=7$ or $4+3=7$, a serum prostate-specific antigen (PSA) between 10-20 $\mathrm{ng} / \mathrm{mL}$ and clinical T2b or T2c disease. The heterogeneity of this patient group is well described, and the risk prediction for an individual patient in this group needs improvement especially if one is to utilize active surveillance as a possible disease management approach for a subset of these patients [8].

To identify the level of genomic instability present in intermediate risk prostate cancer patients' CTCs and to potentially link this genomic hallmark to the risk to disease progression, we applied three-dimensional (3D) fluorescent telomere imaging and profiling to CTCs. 3D telomere profiling is a quantitative assay [17]: it determines the level of ongoing genomic instability by measuring telomere-specific structural genetic parameters of each nucleus as previously reported [18-21]. This includes the measurement of the level of aneuploidy (gain and loss of chromosomes) present in each nucleus using the fluorescent telomere signal numbers as indicators [22,23]. 3D telomere profiling further determines the level of ongoing genomic rearrangements through the measurement of telomeric aggregates (clusters of telomeres) and telomere lengths [24]. Nuclear volumes are also determined and telomere numbers per nuclear volume assessed [17]. The single nucleus readout of 3D telomere measurements therefore is the immediate summary of the genomic instability found in every cell. Moreover, these measurements enable the determination of genomic cell-to-cell heterogeneity [25]. This technology has been previously applied to other cancers and was applied to subgroup patients [26-31].

Using a filtration-based technique (ScreenCell, Paris, France) [32], we have successfully isolated CTCs from different cancers including prostate adenocarcinoma $[25,33]$. We have also shown that this filtration device is suitable to isolate CTCs from all prostate cancer risk groups, including the intermediate risk group of prostate cancer [34].

In this current report, 3D telomere profiling was carried out in a blinded fashion, with 65 treatment-naïve intermediate risk prostate cancer patients consenting to having their blood drawn for the 3D telomere analysis of their CTCs. The study endpoint was the final pathology report after radical prostatectomy (RP). At that time, CTC telomere profiling data and pathology reports were compared, and statistical analyses performed. We report on the ability of 3D telomere profiling to identify patients with stable or aggressive/progressive forms of the disease.

\section{Results}

\subsection{Pathology Reports after Radical Prostatectomy Show Close Association with Pre-Operative 3D Profiling} Results of CTCS

All patients included in our pilot study underwent radical prostatectomy (RP). RP pathology results were compared with the statistical analysis of the 3D telomere profiles obtained from pre-operative 3D telomere profiling of CTCs. Table 1 shows intermediate risk prostate cancer patients who, at RP, had the stable form of the disease when compared to pre-operative biopsy or had disease upgrading. The latter is highlighted in bold in Table 1. The RP reports of the latter patients indicated that the tumor was no 
longer Gleason $7(3+4)$ but displayed a primary pattern 4 or $>$ Gleason 7 , which showed tertiary pattern 5 and/or lymph node involvement.

\subsection{Three-Dimensional (3D) Telomere Profiling of CTCs from Intermediate Risk Prostate Cancer Patients}

Three-dimensional (3D) telomere analysis using TeloView ${ }^{\mathrm{TM}}$ analysis [17] of CTCs from each of the patients provided data for each telomere in each nucleus. Hierarchical centroid cluster analysis was performed with all data sets, and the patients were grouped into three subgroups (clusters 1-3). Figure 1 shows the distribution of the patients after clustering.

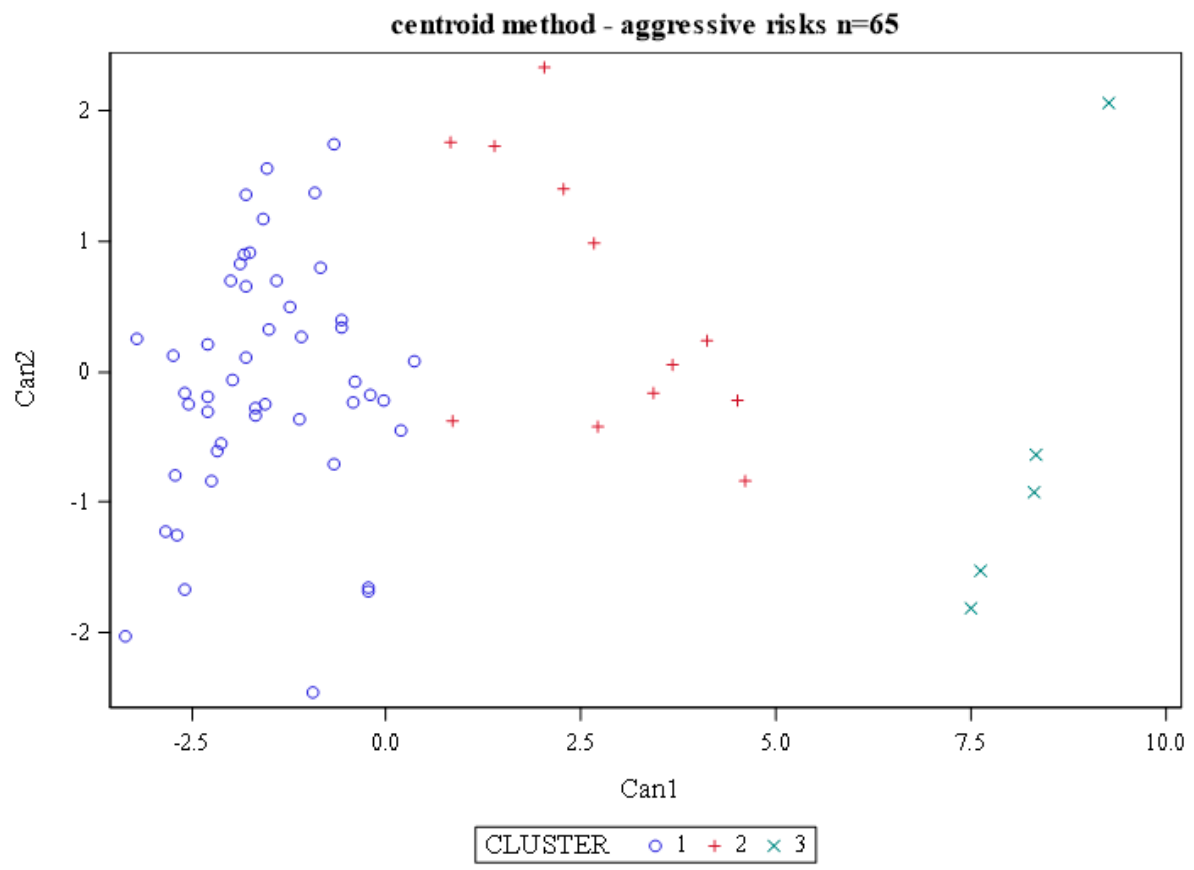

Figure 1. Centroid cluster analysis of 3D nuclear profiling of circulating tumor cells (CTCs) from 65 patients with intermediate risk prostate cancer. A combination of telomere parameters (Materials and Methods) led to the stratification of patients into clusters, each with a different level of genomic instability and a different risk to progression. Patients in cluster 3 (green) had the lowest risk (20\%) to progression, while those in cluster two (red) and cluster 1 (blue) had an intermediate (50\%) to high risk $(68.75 \%)$, respectively.

The 3D telomere profiling of CTCs established a clear association with the RP pathology findings summarized in Table 1. The three clusters identified from 3D telomere profiling data after hierarchical centroid cluster analysis identified patients with different levels of genomic instability and different risk to progression (Figure 1). Cluster 3 contains predominantly stable intermediate risk prostate cancer patients, in which $20 \%$ of the patients have aggressive disease. This cluster has one outlier in the top right corner. This patient's CTCs had comparatively larger nuclei and more telomeres per nuclear volume than the rest of cluster 3 . However, overall, the other telomere parameters placed him in this cluster. In contrast, cluster 2 of intermediate risk prostate cancer includes an intermediate risk group, in which $50 \%$ of the patients represent those with aggressive disease. The higher risk grouping of the intermediate risk prostate cancer group is found in cluster 1 and comprises $68.75 \%$ of the patients in our pilot study. Thus, as patients are observed in clusters 3, 2 or 1, their disease appears more aggressive (cluster $1>$ cluster $2>$ cluster 3 ) based on their genomic instability pattern detected by 3D telomere analysis. 
Table 1. Clinical information of 65 intermediate risk prostate cancer patients at biopsy and after radical prostatectomy. Bold highlights patients whose cancer was upgraded after radical prostatectomy (RP). Days to surgery after circulating tumor cells (CTC) collection varied among patients. The results of the hierarchical centroid cluster analysis (Figure 1) are indicated for each patient (clusters 1, 2 or 3).

\begin{tabular}{|c|c|c|c|c|c|c|c|}
\hline $\begin{array}{l}\text { Patient } \\
\text { Number }\end{array}$ & Gleason & Stage & $\begin{array}{l}\text { Prostate Specific } \\
\text { Antigen (PSA) }\end{array}$ & Cluster & $\begin{array}{l}\text { Days to } \\
\text { Surgery }\end{array}$ & $\begin{array}{l}\text { Gleason } \\
\text { Score }\end{array}$ & Clinical Data \\
\hline \multicolumn{7}{|c|}{ Before Radical Prostatectomy } & After Radical Prostatectomy \\
\hline 1 & $4+3$ & $\mathrm{~T} 2 \mathrm{c}$ & 8.9 & 3 & 1 & $3+4$ & RP 03/2014 (no tert pattern, pT2c N0), PSA currently undetectable \\
\hline 2 & $4+3$ & T1c & 14.71 & 1 & 10 & $4+3$ & $\begin{array}{l}\text { RP 12/2012 (tert } 5 \text { pattern, pT2c N0), PSA currently } \\
\text { undetectable }\end{array}$ \\
\hline 3 & $4+3$ & $\mathrm{~T} 2 \mathrm{~b}$ & 3.26 & 1 & 10 & $4+3$ & $\begin{array}{l}\text { RP 08/2014 (T3b N1, no tert pattern), PSA currently } \\
\text { undetectable }\end{array}$ \\
\hline 4 & $3+4$ & T1c & 4.18 & 1 & 10 & $3+4$ & $\begin{array}{l}\text { RP 2/2018 (tert } 5 \text { pattern, pT2N0), 9/27/18 PSA }<0.01 \text {, PSA } \\
\text { currently undetectable }\end{array}$ \\
\hline 5 & $3+4$ & $\mathrm{~T} 2 \mathrm{a}$ & 8.3 & 2 & 11 & $3+4$ & $\begin{array}{c}\text { RP 10/2014 (no tert pattern, pT3b, N1), BCR, RT + ADTx1yr, } \\
\text { PSA currently undetectable }\end{array}$ \\
\hline 6 & $3+4$ & T1c & 5.7 & 2 & 14 & $3+4$ & RP 08/2012 (no tert pattern, pT2 N0), PSA currently undetectable \\
\hline 7 & $4+3$ & T1c & 3.85 & 2 & 15 & $3+4$ & RP 09/2014 (no tert pattern, pT2c N0), PSA currently undetectable \\
\hline 8 & $3+4$ & T1c & 11.8 & 3 & 16 & $3+4$ & RP 12/2012 (no tert pattern, pT2c N0), PSA currently undetectable \\
\hline 9 & $3+4$ & T1c & 3.5 & 1 & 17 & $3+4$ & $\begin{array}{c}\text { RP 12/2012 (tert } 5 \text { pattern, pT3b N0), PSA currently } \\
\text { undetectable }\end{array}$ \\
\hline 10 & $3+4$ & T1c & 4.93 & 2 & 17 & $3+4$ & $\begin{array}{c}\text { RP 09/2014 (no tert pattern, pT3a N0), PSA currently } \\
\text { undetectable, 9/7/17 PSA }<0.01 .\end{array}$ \\
\hline 11 & $3+4$ & T2a & 9.5 & 2 & 17 & $3+4$ & RP 09/2014 (no tert pattern, pT2c NX), PSA currently undetectable \\
\hline 12 & $3+4$ & T2a & 5 & 1 & 17 & $3+4$ & RP 04/2015 (pT3a N0), PSA currently undetectable \\
\hline 13 & $4+3$ & T1c & 7.86 & 1 & 17 & $4+3$ & $\begin{array}{c}\text { RP 11/2017 (tert } 5 \text { pattern, pT2c N0), 1/2/18 PSA }<0.01 \text {, PSA } \\
\text { currently undetectable }\end{array}$ \\
\hline
\end{tabular}


Table 1. Cont

\begin{tabular}{|c|c|c|c|c|c|c|c|}
\hline $\begin{array}{l}\text { Patient } \\
\text { Number }\end{array}$ & Gleason & Stage & $\begin{array}{l}\text { Prostate Specific } \\
\text { Antigen (PSA) }\end{array}$ & Cluster & $\begin{array}{l}\text { Days to } \\
\text { Surgery }\end{array}$ & $\begin{array}{l}\text { Gleason } \\
\text { Score }\end{array}$ & Clinical Data \\
\hline \multicolumn{7}{|c|}{ Before Radical Prostatectomy } & After Radical Prostatectomy \\
\hline 14 & $\begin{array}{c}4+3 \\
(9 / 2016) \\
4+3 \\
(11 / 2017)\end{array}$ & T1c & $7.22,7.30$ & 1 & 17 & $4+5$ & $\begin{array}{c}\text { RP scheduled 5/2018, (no tert pattern, pT2 N0), 6/19/18 PSA < } \\
\text { 0.01, PSA currently undetectable }\end{array}$ \\
\hline 15 & $4+3$ & T2a & 5 & 1 & 23 & $4+3$ & $\begin{array}{l}\text { RP 10/2014 (no tert pattern, pT3a, invasion of bladder neck } \\
\text { muscle), PSA currently undetectable }\end{array}$ \\
\hline 16 & $3+4$ & T1c & 15 & 1 & 24 & $4+3$ & $\begin{array}{c}\text { RP 04/2015 (tertiary } 5 \text { pattern, T2c N0), PSA currently } \\
\text { undetectable, 11/13/18 PSA }<0.01\end{array}$ \\
\hline 17 & $4+3$ & $\mathrm{~T} 2 \mathrm{~b}$ & 9 & 1 & 26 & $4+3$ & RP 12/2012 (no tert pattern, pT2c N0), PSA currently undetectable \\
\hline 18 & $4+3$ & T1c & 5.56 & 1 & 27 & $4+3$ & RP June 25, 2018 (tert 5 pattern, T3aN1), 8/20/18 PSA $<0.01$ \\
\hline 19 & $\begin{array}{c}3+3 \\
(3 / 2008) \\
3+3 \\
(2 / 2009) \\
\text { benign } \\
(11 / 2011) \\
3+3 \\
(3 / 2014) \\
4+3 \\
(10 / 2017)\end{array}$ & T1c & $\begin{array}{l}3.98,4.97,5.6,4.89 \\
5.71\end{array}$ & 1 & 27 & $4+3$ & $\begin{array}{l}\text { RP June 25, } 2018 \text { (tert } 5 \text { pattern, T3bN0), 8/8/18 PSA 1.71, } \\
\text { 8/28/18 PSA 1.84, ADT Oct } 2018 \text { (plan to be on for 6-8 months), } \\
\text { 10/9/18 PSA } 10.8\end{array}$ \\
\hline 20 & $4+3$ & $\mathrm{~T} 2 \mathrm{a}$ & 3.8 & 1 & 28 & $3+4$ & RP 09/2014 (no tert pattern, pT2c N0), PSA currently undetectable \\
\hline 21 & $3+4$ & T1c & 5 & 1 & 30 & $3+4$ & $\begin{array}{c}\text { RP 06/2013 (no tert pattern, pT3a N0), PSA currently } \\
\text { undetectable }\end{array}$ \\
\hline 22 & $3+4$ & T1c & 8.9 & 1 & 31 & $3+4$ & $\begin{array}{c}\text { RP 09/2014 (tert } 5 \text { pattern, node }+ \text { ve), BC failure, received RT } \\
\text { /ADT } \times 2 \text { yrs, PSA currently undetectable }\end{array}$ \\
\hline 23 & $4+3$ & $\mathrm{~T} 2 \mathrm{c}$ & 2.8 & 3 & 35 & $3+4$ & $\begin{array}{l}\text { RP 10/2014 (no tert pattern, pT2c N1), PSA currently } \\
\text { undetectable, 5/1/18 PSA 0.19, 11/6/18 PSA } 0.23\end{array}$ \\
\hline 24 & $4+3$ & $\mathrm{~T} 2 \mathrm{a}$ & 5.02 & 1 & 36 & $4+3$ & RP 01/2014 (no tert pattern, pT2c N0), PSA currently undetectable \\
\hline
\end{tabular}


Table 1. Cont.

\begin{tabular}{|c|c|c|c|c|c|c|c|}
\hline $\begin{array}{c}\text { Patient } \\
\text { Number }\end{array}$ & Gleason & Stage & $\begin{array}{l}\text { Prostate Specific } \\
\text { Antigen (PSA) }\end{array}$ & Cluster & $\begin{array}{l}\text { Days to } \\
\text { Surgery }\end{array}$ & $\begin{array}{l}\text { Gleason } \\
\text { Score }\end{array}$ & Clinical Data \\
\hline \multicolumn{7}{|c|}{ Before Radical Prostatectomy } & After Radical Prostatectomy \\
\hline 25 & $3+4$ & $\mathrm{~T} 1 \mathrm{c}$ & 27 & 1 & 36 & $3+4$ & $\begin{array}{c}\text { RP 01/2018 (no tert pattern, pT3aN0), 3/7/18 PSA 0.01, PSA } \\
\text { currently undetectable }\end{array}$ \\
\hline 26 & $4+3$ & $\mathrm{~T} 1 \mathrm{c}$ & 16 & 1 & 37 & $4+3$ & $\begin{array}{c}\text { RP 04/2014 (tert } 5 \text { pattern, pT3b N1), ADT } \times 2 \text { yrs, PSA } \\
\text { currently undetectable }\end{array}$ \\
\hline 27 & $4+3$ & T2a & 14.5 & 1 & 38 & $3+4$ & $\begin{array}{l}\text { RP } 11 / 2017 \text { (no tert pattern, p3a N1), 12/18/17 PSA } 0.03,1 / 30 / 18 \\
\text { PSA 0.02, PSA currently undetectable }\end{array}$ \\
\hline 28 & $4+3$ & $\mathrm{~T} 1 \mathrm{c}$ & 7.7 & 1 & 44 & $4+3$ & $\begin{array}{l}\text { RP 08/2012, (no tert pattern, pT3a N1) node + ve, salvage ADT } \\
2013,2 / 5 / 16 \text { PSA } 0.04\end{array}$ \\
\hline 29 & $4+3$ & $\mathrm{~T} 2 \mathrm{a}$ & 8.91 & 2 & 44 & $4+3$ & $\begin{array}{c}\text { RP Aug 9, } 2018 \text { (no tert pattern, T2N0), 10/16/18 PSA 0.01, PSA } \\
\text { currently undetectable }\end{array}$ \\
\hline 30 & $3+4$ & $\mathrm{~T} 1 \mathrm{c}$ & 11.15 & 2 & 45 & $3+4$ & $\begin{array}{l}\text { RP 08/2013, PSA currently undetectable, (no tert pattern T3a } \\
\text { N0 MX), 6/11/18 slow rising PSA } 0.24 \text {, salvage RT 8/2018 }\end{array}$ \\
\hline 31 & $4+3$ & T1c & 5.4 & 1 & 45 & $4+3$ & RP 03/2015 (no tert pattern, pT2c N0), PSA currently undetectable \\
\hline 32 & $3+4$ & $\mathrm{~T} 1 \mathrm{c}$ & 5.88 & 1 & 55 & $3+4$ & $\begin{array}{c}\text { RP 07/2015 (tert } 5 \text { pattern, pT2C, N0), PSA currently } \\
\text { undetectable }\end{array}$ \\
\hline 33 & $4+3$ & T2a & 8.28 & 1 & 65 & $4+3$ & RP June 21, 2018, (no tert pattern, T2N0), 8/28/18 PSA $<0.01$ \\
\hline 34 & $3+4$ & T2a & 1.23 & 1 & 66 & $3+4$ & RP 03/2014, (no tert pattern, pT2c N0), 11/21/17 PSA 0.01 \\
\hline 35 & $4+3$ & T1c & 5.86 & 1 & 71 & $4+3$ & $\begin{array}{c}\text { RP 05/2013 (tert } 5 \text { pattern, pT3 N0), BCR, salvage RT 06/2015, } \\
\text { PSA currently undetectable }\end{array}$ \\
\hline 36 & $4+3$ & T1c & 5.7 & 1 & 73 & $4+3$ & $\begin{array}{c}\text { RP 12/2012 (no tert pattern, pT3a N0), BC failure, salvage RT } \\
\text { 09/2014, PSA currently undetectable, BCR 7/31/17 PSA 0.25, } \\
\text { 11/6/18 PSA } 18.12\end{array}$ \\
\hline
\end{tabular}


Table 1. Cont

\begin{tabular}{|c|c|c|c|c|c|c|c|}
\hline $\begin{array}{l}\text { Patient } \\
\text { Number }\end{array}$ & Gleason & Stage & $\begin{array}{l}\text { Prostate Specific } \\
\text { Antigen (PSA) }\end{array}$ & Cluster & $\begin{array}{l}\text { Days to } \\
\text { Surgery }\end{array}$ & $\begin{array}{l}\text { Gleason } \\
\text { Score }\end{array}$ & Clinical Data \\
\hline \multicolumn{7}{|c|}{ Before Radical Prostatectomy } & After Radical Prostatectomy \\
\hline 37 & $\begin{array}{c}3+3 \\
11 / 2015,4 \\
+3 \\
(4 / 2017)\end{array}$ & T1c & $9.0,13.74$ & 1 & 85 & $3+4$ & $\begin{array}{c}\text { RP 12/2017 (tert } 2 \text { pattern, pT3b N1 MX), 2/27/18 PSA 0.09, } \\
\text { ADT } 6 \text { months 11/5/18 PSA } 0.01\end{array}$ \\
\hline 38 & $4+3$ & T1c & 7.5 & 1 & 86 & $4+3$ & $\begin{array}{c}\text { RP 01/2013 (tert pattern 5, pT2c), PSA currently undetectable, } \\
\text { 1/11/18 PSA } 0.08\end{array}$ \\
\hline 39 & $3+4$ & T1c & 3.71 & 1 & 90 & $3+4$ & RP April 23, 2018 (no tert pattern, pT2 N0), 6/26/18 PSA 0.01 \\
\hline 40 & $3+4$ & T1c & 19.73 & 1 & 99 & $3+4$ & $\begin{array}{c}\text { RP 03/2014 (tert } 5 \text { pattern, pT3aNOMX) PSA is currently } \\
\text { undetectable, 4/4/18 PSA }<0.01\end{array}$ \\
\hline 41 & $3+4$ & T2a & 2.24 & 1 & 101 & $3+4$ & $\begin{array}{l}\text { RP 03/2013 (no tert pattern, pT2c N0, significant volume), PSA } \\
\text { currently undetectable }\end{array}$ \\
\hline 42 & $3+4$ & T1c & 14.51 & 1 & 101 & $3+4$ & $\begin{array}{c}\text { RP 12/2017 (tert } 5 \text { pattern, pT3a N0), 3/27/18 PSA } 0.12 \text { gradually } \\
\text { rising, 10/1/18 PSA 0.25, Adjuvant RT 10/2018 }\end{array}$ \\
\hline 43 & $4+3$ & T1c & 11.53 & 2 & 102 & $3+4$ & $\begin{array}{c}\text { RP 12/2017 (no tert pattern, pT2b N0), 2/22/18 PSA }<0.01 \text {, PSA } \\
\text { currently undetectable }\end{array}$ \\
\hline 44 & $\begin{array}{c}3+4 \\
(3 / 2012) \\
3+4 \\
(1 / 2013) \\
\text { Benign } \\
(9 / 2014) \\
4+3 \\
(9 / 2017)\end{array}$ & T1c & $\begin{array}{l}10.8,12.64,8.99 \\
17.92\end{array}$ & 1 & 121 & $4+3$ & $\mathrm{RP}$ 6/1/18 (no tert pattern, pT2N0), 8/17/18 PSA 0.03 \\
\hline 45 & $4+3$ & T2a & 9.3 & 1 & 128 & $4+4$ & $\begin{array}{l}\text { RP August 9, 2018. (tert } 5 \text { pattern, T3aN1), 10/22/18 PSA 0.77, } \\
\text { ADT received } 1 \text { dose 11/2018 (plan is for 6-8 months), 11/22/18 } \\
\text { PSA } 0.69\end{array}$ \\
\hline
\end{tabular}


Table 1. Cont

\begin{tabular}{|c|c|c|c|c|c|c|c|}
\hline $\begin{array}{l}\text { Patient } \\
\text { Number }\end{array}$ & Gleason & Stage & $\begin{array}{l}\text { Prostate Specific } \\
\text { Antigen (PSA) }\end{array}$ & Cluster & $\begin{array}{l}\text { Days to } \\
\text { Surgery }\end{array}$ & $\begin{array}{l}\text { Gleason } \\
\text { Score }\end{array}$ & Clinical Data \\
\hline \multicolumn{7}{|c|}{ Before Radical Prostatectomy } & After Radical Prostatectomy \\
\hline 46 & $3+4$ & $\mathrm{~T} 2 \mathrm{~b}$ & 3.31 & 1 & 134 & $3+4$ & $\begin{array}{l}\text { RP 1/2018, (no tert pattern, pT2 N0), 2/28/18 PSA }<0.01 \text {, PSA } \\
\text { currently undetectable }\end{array}$ \\
\hline 47 & $4+3$ & $\mathrm{~T} 1 \mathrm{c}$ & 5.5 & 3 & 138 & $4+3$ & $\begin{array}{c}\text { RP April 23, } 2018 \text { (no tert pattern, pT2cN0), 9/4/18 PSA }<0.01 \\
\text { PSA currently undetectable }\end{array}$ \\
\hline 48 & $3+4$ & $\mathrm{~T} 1 \mathrm{c}$ & 4.95 & 1 & 143 & $3+4$ & $\begin{array}{c}\text { RP 09/2013 (pT2c N0, 75\% involvement), PSA currently } \\
\text { undetectable, 6/22/18 PSA 0.14 }\end{array}$ \\
\hline 49 & $4+3$ & $\mathrm{~T} 1 \mathrm{c}$ & 17.08 & 1 & 144 & $3+4$ & $\begin{array}{c}\text { RP 6/15/2018 (no tert pattern, pT2N0MX), 9/18/18 PSA }<0.01 \\
\text { PSA currently undetectable }\end{array}$ \\
\hline 50 & $4+3$ & $\mathrm{~T} 2 \mathrm{a}$ & 10.34 & 2 & 149 & $4+3$ & $\begin{array}{c}\text { RP 11/2012 (tert pattern 5, pT2c N0), PSA currently } \\
\text { undetectable }\end{array}$ \\
\hline 51 & $4+3$ & T1c & 6.51 & 3 & 156 & $4+3$ & RP May 10, 2018, (no tert pattern, pT3aN0), 8/7/18 PSA 0.02 \\
\hline 52 & $4+3$ & $\mathrm{~T} 2 \mathrm{a}$ & 5.61 & 1 & 157 & $4+3$ & $\begin{array}{c}\text { On AS, progression on F/U biopsy, RP } 07 / 2014 \text { (no tert pattern, } \\
\text { pT3a N0), PSA currently undetectable }\end{array}$ \\
\hline 53 & $4+3$ & T1c & 7.88 & 1 & 162 & $4+3$ & RP 7/12/18 (tert 5 pattern, T2N0), 9/26/18 PSA $<0.01$ \\
\hline 54 & $\begin{array}{c}3+3 \\
(12 / 2015) \\
3+3 \\
(1 / 2017) \\
4+3 \\
(12 / 2017\end{array}$ & $\mathrm{T} 1 \mathrm{c}$ & $4.25,12.77,19.49$ & 1 & 166 & & RP Oct 2, 2018 (no tert pattern, T2 N0 MX) \\
\hline 55 & $4+3$ & $\mathrm{~T} 2 \mathrm{a}$ & 1.41 & 1 & 167 & $4+3$ & $\begin{array}{c}\text { RP 09/2013 (tert } 5 \text { pattern, pT3b N1), climbing PSA to 0.14, RT } \\
\text { 04/2015, ADT } \times 2 \text { yrs }\end{array}$ \\
\hline
\end{tabular}


Table 1. Cont

\begin{tabular}{|c|c|c|c|c|c|c|c|}
\hline $\begin{array}{l}\text { Patient } \\
\text { Number }\end{array}$ & Gleason & Stage & $\begin{array}{l}\text { Prostate Specific } \\
\text { Antigen (PSA) }\end{array}$ & Cluster & $\begin{array}{l}\text { Days to } \\
\text { Surgery }\end{array}$ & $\begin{array}{l}\text { Gleason } \\
\text { Score }\end{array}$ & Clinical Data \\
\hline \multicolumn{7}{|c|}{ Before Radical Prostatectomy } & After Radical Prostatectomy \\
\hline 56 & $\begin{array}{c}3+3 \\
(2 / 2017) \\
3+4 \\
(5 / 2017)\end{array}$ & T1c & 18 & 1 & 167 & $4+3$ & ADT 3 months, RP 3/28/18 (tert 5 pattern, pT2cN0), PSA 0.01 \\
\hline 57 & $3+4$ & $\mathrm{~T} 2 \mathrm{a}$ & 9.4 & 2 & 177 & $4+3$ & $\begin{array}{l}\text { On AS, grade/volume progression }(4+3 \text { in } 6 \text { cores, Comedo } \\
\text { necrosis suggestive of pattern 5), RP 02/2013 (no tert pattern, } \\
\text { pT3a N0), PSA currently undetectable }\end{array}$ \\
\hline 58. & $4+3$ & T1c & 12 & 2 & 178 & $3+4$ & RP Oct 11, 2018 (no tert pattern, T3a N0) \\
\hline 59 & $\begin{array}{c}4+3 \\
(11 / 2016) \\
3+4 \\
(1 / 2018)\end{array}$ & T1c & $6.68,7.74$ & 1 & 184 & & RP Nov 22, 2018 (tert 5 pattern, T3b N0) \\
\hline 60 & $4+3$ & T1c & 11.13 & 1 & 204 & $4+3$ & RP 8/23/2018 (tert 5 pattern, T3a N0), 9/24/18 PSA 0.01 \\
\hline 61 & $3+4$ & $\mathrm{~T} 2 \mathrm{~b}$ & 6.7 & 1 & 207 & $3+4$ & RP Nov 5, 2018 (no tert pattern T2 N0) \\
\hline 62 & $3+4$ & T1c & 8.48 & 1 & 209 & $4+5$ & RP Nov 20, 2018 (tert 3 pattern, T2N0MX) \\
\hline 63 & $3+4$ & T1c & 9 & 2 & 225 & $3+4$ & RP 04/2013 (no tert pattern, pT2c N0), PSA currently undetectable \\
\hline 64 & $4+3$ & T1c & 16.55 & 1 & 282 & $4+3$ & RP Oct 19, 2018 (no tert pattern, T3b N1) \\
\hline 65 & $3+4$ & $\mathrm{~T} 2$ & 1.84 & 1 & 317 & $3+4$ & RP 11/29/18 (no tert pattern, T3a N0) \\
\hline
\end{tabular}


Figure 2 illustrates 3D images of telomere parameters for a representative patient of each cluster. The representative images show that the organizational and spatial features of telomeres in the nuclei of CTCs increase in their degree of aberration from cluster 3 to 2 to 1 . The total number of telomeric signals is highest in cluster 1 as are the number of telomeres per nuclear volume and the nuclear volumes (Figure 2). Cluster 1 shows a representative nucleus with a diameter of $20 \mu \mathrm{m}$. Cluster 2 is intermediate between clusters 1 and 3; in this cluster, the numbers of telomeric signals and the numbers of telomeres per nuclear volume are increased compared to cluster 3 but are lower than in cluster 1 . Similarly, the nuclear volumes are increased in cluster 2 compared to cluster 3 (nuclear diameters of $13 \mu \mathrm{m}$ vs. $9 \mu \mathrm{m}$, respectively), but below those seen in cluster 1 (Figure 2). Thus, there is a marked increase in the level of aberrant spatial telomere organization from cluster 3 to 2 to 1 .

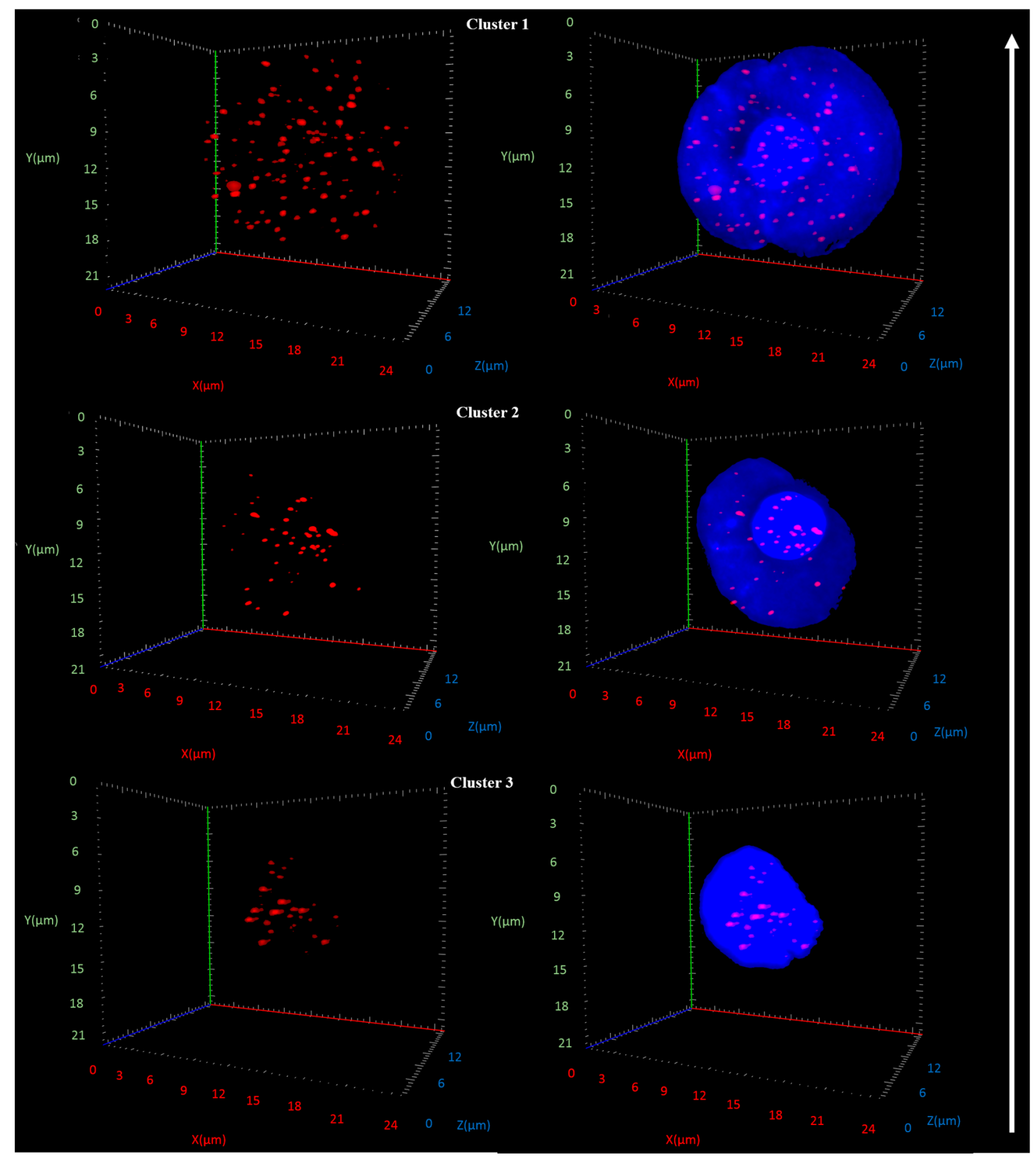

Figure 2. Representative images of CTCs from patients in cluster 1, 2 and 3. Left panels: 3D telomere (red) images. Right panels: 3D telomeres (red) in CTC nuclei (blue). Note the micrometer $(\mu)$ scales for nuclear sizes as shown for each 3D image in the $x, y, z$ positions of the image. A white arrow indicates the increase in the level of genomic instability from cluster 3 to 2 to 1 (for details, see text). 


\subsection{Modeling of 3D Telomeric CTC Parameters}

We next performed logistic regression modeling using the 3D telomere parameters measured by TeloView [17] (File S1). Logistic regression modeling showed that four parameters separated stable from aggressive disease (Figure 3). At $95 \%$ confidence levels, odds ratios of $<1$ are indicative of stable disease; odds ratios $>1$ are indicative of aggressive disease. The following $3 \mathrm{D}$ telomere parameters separated the stable from aggressive disease, nuclear volume (nucvolp10kiqr), telomeres per nuclear volume (telp100kv25), total number of signals (tnsignal25), and nuclear volume (nucv175p10k).

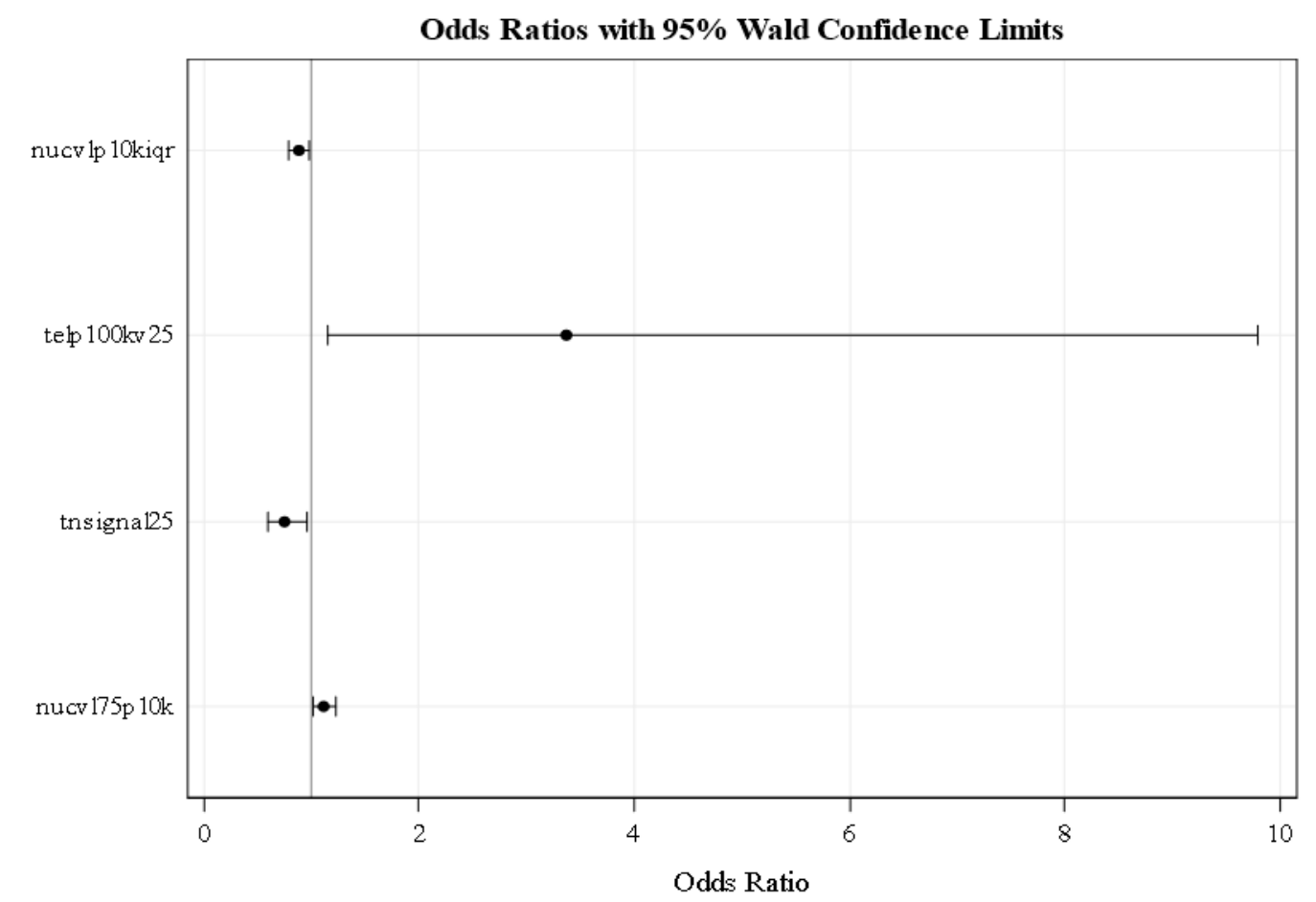

Figure 3. Logistic regression modeling identified four parameters that enable the distinction of stable vs. aggressive disease as measured by $3 \mathrm{D}$ telomere profiling in patients with intermediate risk prostate cancer. At a confidence level of $95 \%$, patients with values $<1$ are considered to be with stable disease at the time point of the analysis, while patients with values $>1$ are considered to be with aggressive disease. The wide 95\% confidence interval (CI) observed for telp100kv25 indicates that this predictor is less accurate than the other three that all display a very narrow $95 \%$ CI. Abbreviations in this figure: iqr: interquartile range (the difference between the 75th percentile and the 25th percentile); nucvolp10kiqr: nuclear volume $(10 \times$ interquartile); telp100kv25: telomeres per nuclear volume $(100 \times$ nuclear volume 25th quartile), tnsignal25: total number of telomere signals (25th quartile), nucvol75p10k: nuclear volume $(10 \times 75$ th quartile $)$.

These four predictors were modeled and resulted in a receiver operating characteristic (ROC) curve for this pilot study (Figure 4). For 3D telomere profiling of pre-operative CTCs, the area under the curve (AUC) was 0.77 , and a sensitivity of $75 \%$ and specificity of $68 \%$ were reached (Figure $4 \mathrm{~A}$ ). In contrast, pre-operative PSA of these patients reached an AUC of 0.59 (Figure 4B).

Time to RP varied among the patients included in this study (Section Materials and Methods, Table 1). Patients $\leq 3$ months showed the best predicted linear trend, with one degree Mantel-Haenszel chi-sqared (trend test), $p=0.008,75 \%$ sensitivity, and $73.33 \%$ specificity. Patients $\leq 6$ months had better sensitivity $(77.14 \%)$ but poorer specificity $(65.22 \%)$. Taken together, the whole group of patients showed a sensitivity of $75 \%$ and specificity of $68 \%$. A future and larger cohort study should potentially focus on patients $\leq 3$ months of RP, which may further improve predictability. 


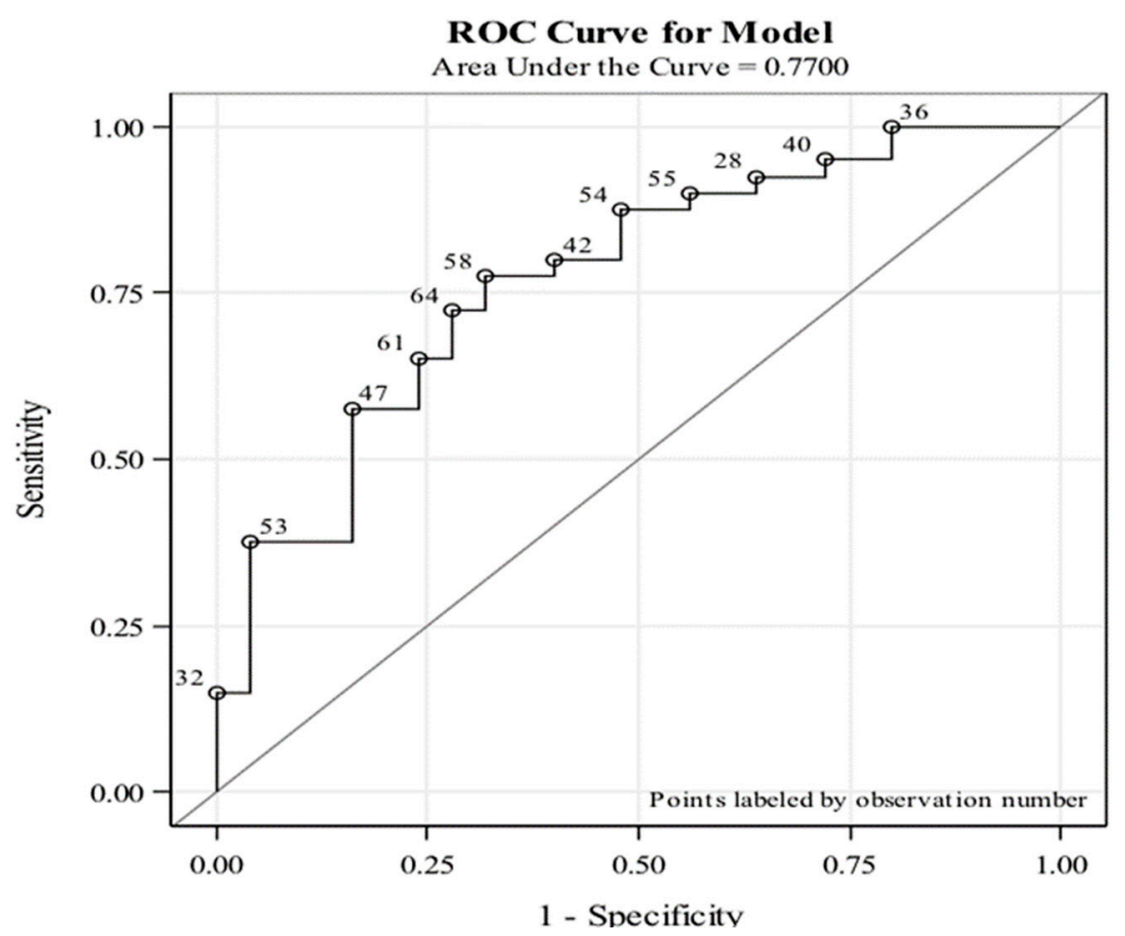

(A)

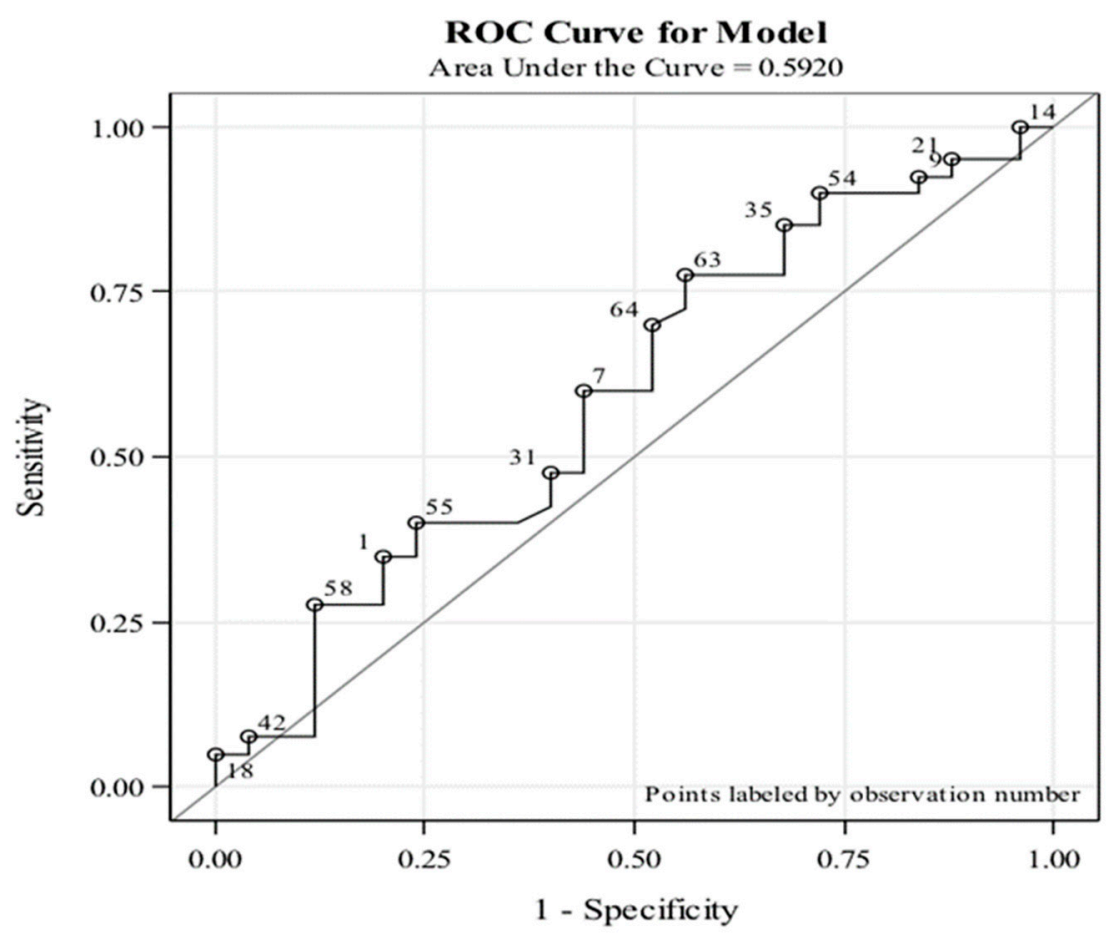

(B)

Figure 4. Receiver operating curve (ROC) for 3D telomere analysis of pre-operative CTCs from 65 intermediate risk prostate cancer patients compared to their prostate specific antigen (PSA) values. (A): ROC curve for 3D telomere profiling of CTCs. (B): ROC curve for PSA. The y axes display the sensitivity and the $x$ axes display the specificity of the assays. 


\section{Discussion}

Intermediate risk prostate cancer represents a challenge to patients and clinicians as this group is heterogenous and the risk to progression unclear. Current PSA screening and biopsies that give Gleason scores $(3+4$ or $4+3)$ do not suffice in predicting disease progression [1,5]. As active surveillance is more readily utilized for low tier intermediate risk patients with $3+4$ disease, reliability regarding risk stratification is desperately needed. The need for better identification/stratification of indolent or more aggressive disease motivated this study.

To this end, we examined pre-operative CTCs isolated from the blood of 65 intermediate risk prostate cancer patients. 3D telomere profiling was used to assess the level of genomic instability present in the CTCs of each patient. The study was done in a blinded manner. After completion of analysis, final pathology results of RPs became available and patients were identified with "stable" or "upgraded" disease status (Table 1). Forty of the 65 patients were upgraded based on the RP pathology results.

3D telomere profiling as utilized in this report, has previously been applied to other cancers, including neuroblastoma, glioblastoma, myelodysplastic syndromes and acute myeloid leukemia, Hodgkin's lymphoma, multiple myeloma, and thyroid cancer [8-13]. In all cases examined so far, this technology was able to identify patient subgroups.

This current study is, to our knowledge, the first one applying 3D telomere profiling of CTCs to the heterogeneous intermediate risk prostate cancer patients.

Our 3D telomere data relate to the genomic heterogeneity in prostate cancer patients with Gleason 6 and 7 that was reported by Lalonde et al. [1]. Based on genetic profiling, these authors identified four groups of patients with Gleason 6 and 7. Group 4 consisted of patients with so-called quiet genomes due to few genomic alterations. Patients in the latter subgroup had a significantly better prognosis than those in subtypes 1-3. This study also indicated that the four genomic instability-derived subtypes were independent of Gleason score, T category, and prostate PSA in all cohorts. Individual Gleason 6 tumors had a higher percentage of genome alteration than some Gleason $7(4+3)$ tumors. The percentage of genome alteration was strongly prognostic and independent of clinical covariates. Similarly, Boutros et al. [5] found a high level of heterogeneity in Gleason 7 patients based on copy number variations.

The data obtained with our pilot cohort suggest that 3D telomere profiling is able to stratify intermediate risk prostate cancer patients into risk subgroups. The subgrouping identifies patients with low, intermediate and higher risk of progression as indicated by the centroid cluster analysis of the 3D parameters measured with CTCs. The different levels of genomic instability found in the three clusters correlates with low, medium or elevated risk to progression. Cluster 3 seems to be similar to group 4 in Lalonde's study, while clusters 2 and 1 appear to correspond to the more aggressive phenotypes of the other subtypes [1]. Of note, our study was based on CTCs, while theirs was based on image-guided biopsies.

In contrast to the cluster analysis that used a combination of 3D telomere parameters, logistic regression modeling identified four 3D telomere parameters that separated patients with aggressive/progressive vs. stable disease at confidence interval (CI) of $95 \%$. ROC curves of 3D telomere profiles obtained from pre-operative CTCs were then created based on these four parameters.

$3 \mathrm{D}$ telomere profiling in this pilot cohort provided better reads than the ones obtained with 2600 men in a large study focused on PSA levels [35]. In this former study, the AUC was 0.67 [18]. Other studies on PSA found AUCs of 0.55-0.70 (reviewed in Prensner et al. [36]). In the large study reported by Hoffman et al. [35], PSA of $4 \mathrm{ng} / \mathrm{ml}$ had a sensitivity of $86 \%$ and a specificity of $33 \%$, while PSA of $7 \mathrm{ng} / \mathrm{mL}$ had a sensitivity of $32 \%$ and a specificity of $56 \%$.

The area under the curve AUC we obtained using 3D telomere profiling of pre-operative CTCs of 65 men was 0.77 . For patients $\leq 3$ months, the assay reached $75 \%$ sensitivity and $73.33 \%$ specificity. Patients $\leq 6$ months had better sensitivity $(77.14 \%)$ but poorer specificity $(65.22 \%)$. Taking all patients together irrespective of time to surgery, 3D nuclear telomere profiling reached a sensitivity of $75 \%$ 
and specificity of $68 \%$. PSA for our cohort had an AUC of 0.59 . Gleason $3+4=7$ vs. $4+3=7$ was unable to predict progressive or stable disease $(p>0.6)$. Thus, similar to previous studies in the same intermediate prostate cancer risk group, PSA and Gleason score are less likely to indicate a patient's risk to progression than genetic profiling or 3D structural telomere analysis [1,5].

Admittedly, our sample size was small, and therefore future studies should involve a larger cohort and focus on blood and CTC collection from intermediate risk prostate cancer patients $\leq 3$ months of RP for the best predictive value to be obtained.

These first data on 3D telomere profiling of intermediate risk prostate cancer patients' CTCs demonstrate that CTCs contain potentially useful structural genetic information on the risk of prostate cancer progression. This information cannot be gleaned solely from surgical pathology biopsy specimens as important pathological information is not available on original biopsy due to sampling limitations. In our study, 40 of 65 patients were upgraded after RP and needed RP. In contrast, the remaining 25 patients could have been monitored by active surveillance. The stratification of intermediate risk prostate cancer patients into those with indolent or aggressive disease may be predictable based on pre-operative CTC analysis. The information obtained through 3D CTC telomere profiling could have value in pre-treatment discussions surrounding active surveillance and neo-adjuvant and adjuvant therapy provision.

\section{Materials and Methods}

\subsection{Patients and Collection of Circulating Tumor Cells}

Sixty-five treatment-naïve patients with biopsy-confirmed D'Amico-defined intermediate risk prostate cancer consented to the analysis of their CTCs. Time to RP varied among the patients included in this study. Fifty-eight patients $(89.23 \%)$ were within six months to surgery. Thirty-nine patients $(60 \%)$ were within three months. Two patients were >eight months prior to RP. The patient characteristics before RP and at RP are summarized in Table 1. Ethics Board approval and informed consent was obtained for the study (HS14085, H2011:336).

A total of $9 \mathrm{~mL}$ of blood was collected once per patient pre-RP in Vacutainer ${ }^{\circledR}$ blood collection tubes (with EDTA as an anti-coagulant) and processed within two hours. Patient blood was processed using the ScreenCell ${ }^{R}$ filter method for the separation of prostate CTCs [32]. Briefly, patient blood ( $3 \mathrm{~mL}$ ) was pre-cleared with red blood cells lysis buffer ( 8 mins and $4 \mathrm{~mL}$ ), and the remaining blood cells were prefixed prior to ScreenCell ${ }^{\circledR}(7.50 \pm 0.36 \mu \mathrm{m}$ pore size) filtration to collect cells, which were unable to pass through the filter pores.

\subsection{Three-Dimensional Telomere Hybridization and Analysis}

\subsubsection{Quantitative Fluorescent in Situ Hybridization (QFISH)}

For QFISH, cells on the filters were incubated in $1 \times$ PBS for 5 min followed by a 10 min fixation in $3.7 \%$ formaldehyde $1 \times$ PBS and three washes in $1 \times$ PBS for 5 min each. Filters were treated with $50 \mu \mathrm{g} / \mathrm{mL}$ pepsin (Sigma) in $0.01 \mathrm{M} \mathrm{HCl}$ for $10 \mathrm{~min}$ at $37^{\circ} \mathrm{C}, 1 \times$ washed in $1 \times$ PBS for $5 \mathrm{~min}$ followed by post-fixation for $10 \mathrm{~min}$ in $3.7 \%$ formaldehyde/ $1 \times$ PBS and $3 \times$ washes in $1 \times$ PBS for 5 min each. Filters were dehydrated in an ethanol series $(70 \%, 90 \%$, and 100\% ethanol for 3 min each) and air-dried. Cyanine 3 (Cy3)-conjugated telomere peptide nucleic acid (PNA) probe (DAKO, Glostrup, Denmark) was applied ( $5 \mu \mathrm{L}$ probe/slide), and following denaturation at $80{ }^{\circ} \mathrm{C}$ for $3 \mathrm{~min}$, and hybridization was done for $2 \mathrm{~h}$ at $30^{\circ} \mathrm{C}$. Slides were washed in $70 \%$ deionized formamide (Sigma, Oakville, ON, Canada) in $10 \mathrm{mM}$ Tris pH 7.4 for $15 \mathrm{~min}$, rinsed in $1 \times$ PBS and followed $1 \times$ by $2 \times$ saline-sodium citrate (SSC) $\left(5 \mathrm{~min}\right.$ at $\left.55^{\circ} \mathrm{C}\right), 0.1 \times \mathrm{SSC}$, and $2 \times \mathrm{SSC} / 0.05 \%$ Tween-20 at room temperature. Filters were again dehydrated in a series of ethanol washes (70\%, 90\%, 100\%) and air-dried. Filters were removed from the metal support ring using an $8 \mathrm{~mm}$ biopsy punch, placed 
on a new slide, 4',6-diamidino-2-phenylindole (DAPI)- stained, mounted with Vectashield (Vector Laboratories, Burlington, ON, Canada), and a coverslip.

\subsubsection{Three-Dimensional (3D) Telomere Imaging and Analysis}

Slides were imaged on a Zeiss AxioImager Z2 microscope with a Zeiss AxioCam MRmm Rev 3 digital camera using AxioVision Release 4.8.2 (Zeiss, Jena, Germany). A Cy3 filter was used to detect the Cy3 probe nuclear hybridization to telomeric repeats at an exposure time of $546 \mathrm{~ms}$. Exposure times for the DAPI filter differed between slides. Eighty focal planes spaced $200 \mathrm{~nm}$ apart were imaged to create three-dimensional nuclear images of the circulating tumor cells and lymphocytes on the filter. Images were deconvolved using a constrained iterative algorithm [37]. For each patient, we examined thirty CTCs and performed 3D telomere hybridizations, 3D quantitative imaging, and analysis of 3D telomere parameters using TeloView ${ }^{\mathrm{TM}}$ as described previously $[17,25,34]$. TeloView ${ }^{\mathrm{TM}}$ is proprietary to Telo Genomics (Toronto ON, Canada) and was used with the company's permission. The program measures telomere signals and their intensities, the presence of telomere clusters (aggregates) that, at $200 \mathrm{~nm}$ optical resolution, cannot be further resolved into individual signals, nuclear volumes, and telomeres per nuclear volume. An additional feature of TeloView ${ }^{\mathrm{TM}}$ analysis uses the $\mathrm{a} / \mathrm{c}$ ratio to assess the cell cycle distribution as being G0/G1, S or G2 [17,26]. As the CTCs are collected on filters under mild vacuum, the overall nuclear distribution of telomeres within the oblate spheroid of the nucleus measured through the axes $a, b$, and $c$ is not identical to their distribution in cells examined in the absence of vacuum. Therefore, the $\mathrm{a} / \mathrm{c}$ ratio was not determined in the current study as also previously reported [25]. All other telomere parameters were measured.

\subsection{Statistical Analyses}

T-test and generalized linear model (GLM) procedures did not suffice to identify a single predictor stratifying the patients.

Hierarchical centroid cluster analysis identified three clusters of patients. The clusters were formed by combinations of 3D telomere parameters. Nuclear volume (nuclear volume interquartiles (nucvoliqr)), telomeres per nuclear volume (telomeres per nuclear volume, 25th quartile (telpkvol25)), and percentage of great intensities (greatintpct) were best able to stratify patients into three clusters.

Logistical regression modeling assessed the probability of a patient to fall into the "stable" or "aggressive" group. The following parameters remained at the end of the backward stepwise modeling process: nuclear volume $(10 \times$ inter quartiles (nucvolp10kiqr)), telomeres per nuclear volume $(100 \times$ nuclear volume 25 th quartile (telp100kv25)), total number of telomere signals (25th quartile (tnsignal25)), and nuclear volume $(10 \times 75$ th quartile (nucvl75p10k)). Adjusted odds ratios with $95 \%$ confidence level were estimated. Adjusted odds ratios below 1 were indicative of stable, while odds ratios above 1 were indicative of aggressive disease.

A ROC curve was constructed for the four-parameter telomere model to indicate the concordance between the model and the data. A ROC curve was also constructed for PSA.

\section{Conclusions}

Quantitative 3D telomere profiling allowed for the stratification of the intermediate risk prostate cancer patients into three subgroups, each of which contained patients with different risk to progression. Moreover, logistic regression modeling, identified 3D telomere parameters that separated patients into those with currently stable or progressive disease (with CI of 95\%). 3D telomere profiling data therefore suggest that CTCs contain structural genetic information on the risk of progression of intermediate risk prostate cancer patients. This information is not available in original biopsies because of sampling limitations. Thus, a patient's risk may be predictable based on pre-operative CTC analysis. This information would be highly relevant to pre-treatment discussions surrounding active surveillance and neo-adjuvant and adjuvant therapy provisions. 
Supplementary Materials: The following are available online at http://www.mdpi.com/2072-6694/11/6/855/s1, File S1: TeloView results from each of the 65 patients.

Author Contributions: The design and conceptualization of the study was by S.M., D.D. and J.S. D.D. provided patient samples and clinical input, read, reviewed and edited the manuscript, and co-supervised J.A.A. and A.R.P. J.A.A. performed the experiments. A.R.P. selected images and provided Figure 2 for this article, read, and edited the manuscript. J.S. provided patient samples and clinical input, read, reviewed and edited the manuscript, and co-supervised J.A.A. and A.R.P. D.D. provided patient samples and clinical input, read, reviewed and edited the manuscript, and co-supervised J.A.A. and A.R.P. J.A.A. performed the experiments. A.R.P. selected images and provided Figure 2 for this article, read and edited the manuscript. J.S. provided patient samples and clinical input, read, reviewed and edited the manuscript, and co-supervised J.A.A. and A.R.P. S.M. wrote the manuscript and co-supervised J.A.A. and A.R.P.

Funding: We express our gratitude to the Prostate Cancer Fight Foundation/Manitoba Motorcycle Ride for Dad for financial support of this study (grant number: 76113 6944).

Acknowledgments: The authors thank Mary Cheang (senior system analyst) for statistical analysis, and Adam Yan and Nidhi Shah for their bench work during the summer. We thank Daniel Lichtensztejn for his help with supplemental information and Table 1.

Conflicts of Interest: SM co-founded Telo Genomics Corp, is a shareholder, Director, and Chair of the Clinical and Scientific Advisory Board of the Company. The other authors declare no conflicts of interest.

\section{References}

1. Lalonde, E.; Ishkanian, A.S.; Sykes, J.; Fraser, M.; Ross-Adams, H.; Erho, N.; Dunning, M.J.; Halim, S.; Lamb, A.D.; Moon, N.C.; et al. Tumour genomic and microenvironmental heterogeneity for integrated prediction of 5-year biochemical recurrence of prostate cancer: A retrospective cohort study. Lancet Oncol. 2014, 15, 1521-1532. [CrossRef]

2. Barbieri, C.E.; Demichelis, F.; Rubin, M.A. Molecular genetics of prostate cancer: emerging appreciation of genetic complexity. Histopathology 2012, 60, 187-198. [CrossRef] [PubMed]

3. Beltran, H.; Yelensky, R.; Frampton, G.M.; Park, K.; Downing, S.R.; MacDonald, T.Y.; Jarosz, M.; Lipson, D.; Tagawa, S.T.; Nanus, D.M.; et al. Targeted next-generation sequencing of advanced prostate cancer identifies potential therapeutic targets and disease heterogeneity. Eur. Urol. 2013, 63, 920-926. [CrossRef] [PubMed]

4. Schoenborn, J.R.; Nelson, P.; Fang, M. Genomic profiling defines subtypes of prostate cancer with the potential for therapeutic stratification. Clin. Cancer Res. 2013, 19, 4058-4066. [CrossRef] [PubMed]

5. Boutros, P.C.; Fraser, M.; Harding, N.J.; de Borja, R.; Trudel, D.; Lalonde, E.; Meng, A.; Hennings-Yeomans, P.H.; McPherson, A.; Sabelnykova, V.Y.; et al. Spatial genomic heterogeneity within localized, multifocal prostate cancer. Nat. Genet. 2015, 47, 736-745. [CrossRef]

6. Klotz, L. Contemporary approach to active surveillance for favorable risk prostate cancer. Asian J. Urol. 2019, 6, 146-152. [CrossRef]

7. Kane, C.J.; Eggener, S.E.; Shindel, A.W.; Andriole, G.L. Variability in Outcomes for Patients with Intermediate-risk Prostate Cancer (Gleason Score 7, International Society of Urological Pathology Gleason Group 2-3) and Implications for Risk Stratification: A Systematic Review. Eur. Urol. Focus. 2017, 3, 487-497. [CrossRef]

8. Klotz, L. Active surveillance for intermediate risk prostate cancer. Curr. Urol. Rep. 2017, 18, 80. [CrossRef]

9. Pollard, M.E.; Hobbs, A.R.; Kwon, Y.S.; Katsigeorgis, M.; Lavery, H.J.; Levinson, A.; Bernstein, A.N.; Collingwood, S.A.; Hall, S.J.; Samadi, D.B.; et al. Heterogeneity of Outcomes in D'Amico Intermediate-Risk Prostate Cancer Patients after Radical Prostatectomy: Influence of Primary and Secondary Gleason Score. Oncol. Res. Treat. 2017, 40, 508-514. [CrossRef]

10. Pantel, K.; Hille, C.; Scher, H.I. Circulating Tumor Cells in Prostate Cancer: From Discovery to Clinical Utility. Clin. Chem. 2019, 65, 87-99. [CrossRef]

11. Mai, S. Genomic instability and circulating tumor cells in prostate cancer. Transl. Cancer Res. 2018, 7, S192-S196. [CrossRef]

12. Mader, S.; Pantel, K. Liquid Biopsy: Current Status and Future Perspectives. Oncol. Res. Treat. 2017, 40, 404-408. [CrossRef] [PubMed] 
13. Scher, H.I.; Lu, D.; Schreiber, N.A.; Louw, J.; Graf, R.P.; Vargas, H.A.; Johnson, A.; Jendrisak, A.; Bambury, R.; Danila, D.; et al. Association of AR-V7 on Circulating Tumor Cells as a Treatment-Specific Biomarker With Outcomes and Survival in Castration-Resistant Prostate Cancer. JAMA Oncol. 2016, 2, 1441-1449. [CrossRef] [PubMed]

14. Heller, G.; McCormack, R.; Kheoh, T.; Molina, A.; Smith, M.R.; Dreicer, R.; Saad, F.; de Wit, R.; Aftab, D.T.; Hirmand, M.; et al. Circulating Tumor Cell Number as a Response Measure of Prolonged Survival for Metastatic Castration-Resistant Prostate Cancer: A Comparison With Prostate-Specific Antigen Across Five Randomized Phase III Clinical Trials. J. Clin. Oncol. 2018, 36, 572-580. [CrossRef]

15. Lorente, D.; Olmos, D.; Mateo, J.; Dolling, D.; Bianchini, D.; Seed, G.; Flohr, P.; Crespo, M.; Figueiredo, I.; Miranda, S.; et al. Circulating tumour cell increase as a biomarker of disease progression in metastatic castration-resistant prostate cancer patients with low baseline CTC counts. Ann. Oncol. 2018, 29, 1554-1560. [CrossRef] [PubMed]

16. Scher, H.I.; Graf, R.P.; Schreiber, N.A.; McLaughlin, B.; Lu, D.; Louw, J.; Danila, D.C.; Dugan, L.; Johnson, A.; Heller, G.; et al. Nuclear-specific AR-V7 Protein Localization is Necessary to Guide Treatment Selection in Metastatic Castration-resistant Prostate Cancer. Eur. Urol. 2017, 71, 874-882. [CrossRef] [PubMed]

17. Vermolen, B.J.; Garini, Y.; Mai, S.; Mougey, V.; Fest, T.; Chuang, T.C.; Chuang, A.Y.; Wark, L.; Young, I.T. Characterizing the three-dimensional organization of telomeres. Cytometry A. 2005, 67, 144-150. [CrossRef]

18. Mai, S.; Garini, Y. Oncogenic remodeling of the three-dimensional organization of the interphase nucleus: c-Myc induces telomeric aggregates whose formation precedes chromosomal rearrangements. Cell Cycle 2005, 4, 1327-1331. [CrossRef]

19. Mai, S.; Garini, Y. The significance of telomeric aggregates in the interphase nuclei of tumor cells. J. Cell Biochem. 2006, 97, 904-915. [CrossRef]

20. Gadji, M.; Vallente, R.; Klewes, L.; Righolt, C.; Wark, L.; Kongruttanachok, N.; Knecht, H.; Mai, S. Nuclear remodeling as a mechanism for genomic instability in cancer. Adv. Cancer Res. 2011, 112, 77-126.

21. Knecht, H.; Mai, S. 3D imaging of telomeres and nuclear architecture: An emerging tool of 3D nano-morphology-based diagnosis. J. Cell Physiol. 2011, 226, 859-867. [CrossRef]

22. Mai, S. Initiation of telomere-mediated chromosomal rearrangements in cancer. J. Cell Biochem. 2010, 109, 1095-1102. [CrossRef] [PubMed]

23. Mai, S. The three-dimensional cancer nucleus. Genes Chromosomes Cancer 2019, 58, 462-473. [CrossRef] [PubMed]

24. Louis, S.F.; Vermolen, B.J.; Garini, Y.; Young, I.T.; Guffei, A.; Lichtensztejn, Z.; Kuttler, F.; Chuang, T.C.; Moshir, S.; Mougey, V.; et al. c-Myc induces chromosomal rearrangements through telomere and chromosome remodeling in the interphase nucleus. Proc. Natl. Acad. Sci. USA 2005, 102, 9613-9618. [CrossRef] [PubMed]

25. Adebayo Awe, J.; Xu, M.C.; Wechsler, J.; Benali-Furet, N.; Cayre, Y.E.; Saranchuk, J.; Drachenberg, D.; Mai, S. Three-Dimensional Telomeric Analysis of Isolated Circulating Tumor Cells (CTCs) Defines CTC Subpopulations. Transl. Oncol. 2013, 6, 51-65. [CrossRef]

26. Gadji, M.; Fortin, D.; Tsanaclis, A.M.; Garini, Y.; Katzir, N.; Wienburg, Y.; Yan, J.; Klewes, L.; Klonisch, T.; Drouin, R.; et al. Three-dimensional nuclear telomere architecture is associated with differential time to progression and overall survival in glioblastoma patients. Neoplasia 2010, 12, 183-191. [CrossRef] [PubMed]

27. Gadji, M.; Adebayo Awe, J.; Rodrigues, P.; Kumar, R.; Houston, D.S.; Klewes, L.; Dièye, T.N.; Rego, E.M.; Passetto, R.F.; de Oliveira, F.M.; et al. Profiling three-dimensional nuclear telomeric architecture of myelodysplastic syndromes and acute myeloid leukemia defines patient subgroups. Clin. Cancer Res. 2012, 18, 3293-3304. [CrossRef]

28. Knecht, H.; Sawan, B.; Lichtensztejn, D.; Lemieux, B.; Wellinger, R.J.; Mai, S. The 3D nuclear organization of telomeres marks the transition from Hodgkin to Reed-Sternberg cells. Leukemia 2009, 23, 565-573. [CrossRef]

29. Klewes, L.; Vallente, R.; Dupas, E.; Brand, C.; Grün, D.; Guffei, A.; Sathitruangsak, C.; Awe, J.A.; Kuzyk, A.; Lichtensztejn, D.; et al. Three-dimensional Nuclear Telomere Organization in Multiple Myeloma. Transl. Oncol. 2013, 6, 749-756. [CrossRef]

30. Kuzyk, A.; Gartner, J.; Mai, S. Identification of Neuroblastoma Subgroups Based on Three-Dimensional Telomere Organization. Transl. Oncol. 2016, 9, 348-356. [CrossRef]

31. Caria, P.; Dettori, T.; Frau, D.V.; Lichtenzstejn, D.; Pani, F.; Vanni, R.; Mai, S. Characterizing the three-dimensional organization of telomeres in papillary thyroid carcinoma cells. J. Cell Physiol. 2019, 234, 5175-5185. [CrossRef] 
32. Desitter, I.; Guerrouahen, B.S.; Benali-Furet, N.; Wechsler, J.; Jänne, P.A.; Kuang, Y.; Yanagita, M.; Wang, L.; Berkowitz, J.A.; Distel, R.J.; et al. A new device for rapid isolation by size and characterization of rare circulating tumor cells. Anticancer Res. 2011, 31, 427-441. [PubMed]

33. Wark, L.; Klonisch, T.; Awe, J.; LeClerc, C.; Dyck, B.; Quon, H.; Mai, S. Dynamics of three-dimensional telomere profiles of circulating tumor cells in patients with high-risk prostate cancer who are undergoing androgen deprivation and radiation therapies. Urol. Oncol. 2017, 35, e1-e112. [CrossRef]

34. Awe, J.A.; Saranchuk, J.; Drachenberg, D.; Mai, S. Filtration-based enrichment of circulating tumor cells from all prostate cancer risk groups. Urol. Oncol. 2017, 35, 300-309. [CrossRef] [PubMed]

35. Hoffman, R.M.; Gilliland, F.D.; Adams-Cameron, M.; Hunt, W.C.; Key, C.R. Prostate-specific antigen testing accuracy in community practice. BMC Fam. Pract. 2002, 3, 19. [CrossRef]

36. Prensner, J.R.; Rubin, M.A.; Wei, J.T. Chinnaiyan AM. Beyond PSA: the next generation of prostate cancer biomarkers. Sci. Transl. Med 2012, 4, 127rv3. [CrossRef]

37. Schaefer, L.H.; Schuster, D.; Herz, H. Generalized approach for accelerated maximum likelihood based image restoration applied to three-dimensional fluorescence microscopy. J. Microsc. 2001, 204, 99-107. [CrossRef]

(C) 2019 by the authors. Licensee MDPI, Basel, Switzerland. This article is an open access article distributed under the terms and conditions of the Creative Commons Attribution (CC BY) license (http://creativecommons.org/licenses/by/4.0/). 